Revista Multidisciplinar do Nordeste Mineiro, v.2 2019/02

ISSN 2178-6925

\title{
HIV/AIDS: A IMPORTÂNCIA DO FARMACÊUTICO NA ADESÃO AO TRATAMENTO
}

\section{HIV/AIDS: THE IMPORTANCE OF THE PHARMACIST IN ACCESSION TO TREATMENT}

\begin{abstract}
Raquel Martins Assis
Bacharel em Farmácia. Alfa - Faculdade de Almenara, Almenara, Minas Gerais, Brasil. E-mail: raquelassis2394@gmail.com

Patrícia Alves Cardoso

Mestre. Alfa - Faculdade de Almenara, Almenara, Minas Gerais, Brasil. E-mail: patriciacardosorib@yahoo.com.br

Viviane Amaral Toledo Coelho, Doutora. Alfa - Faculdade de Almenara, Almenara, Minas Gerais, Brasil. E-mail: vivianeatc@yahoo.com.br.
\end{abstract}

Ana Valéria Sousa Campos Pimenta Especialista. Alfa - Faculdade de Almenara, Almenara, Minas Gerais, Brasil. E-mail: anavaleriapimenta@gmail.com

\section{Resumo}

Introdução: A AIDS é uma doença multifacetada e fatores de diferentes ordens produzem situações específicas no Brasil. Objetivo: Apresentar sobre a importância do profissional farmacêutico na adesão ao tratamento da HIV/AIDS. Metodologia: Foi realizada uma revisão de literatura, com natureza exploratória para aprofundar os conhecimentos sobre o tema trabalhado na pesquisa, buscando proporcionar maior familiaridade com o problema. Resultados: O direito ao tratamento dessas pessoas, tal como o de todos os cidadãos, está garantido pela Constituição Federal, sendo responsabilidade do Estado brasileiro. O surgimento de novas formas de tratamento mudou muito o cenário da doença com a diminuição da mortalidade, aumento da expectativa de vida das pessoas que vivem com AIDS. Considerações Finais: Sabe-se que as novas drogas para o tratamento mudaram a história da doença e hoje a AIDS é tratada de forma controlada e fornece segurança ao portador, sendo possível oferecer um combinado de medicamentos, para o tratamento do paciente, sendo possível oferecer harmonia entre a doença e a pessoa infectada. Entretanto, para se alcançar os benefícios do tratamento, é preciso o uso correto da medicação, tanto para início, como para a sua retirada. Portanto, o papel do farmacêutico na assistência a portadores de HIV é essencial para o sucesso do tratamento.

Palavras-Chave: AIDS; Medicamentos; Tratamento; Atenção Farmacêutica.

\section{Abstract}


Introduction: AIDS is a multifaceted disease and factors of different orders produce specific situations in Brazil. Objective: To present the importance of the pharmaceutical professional in adhering to the treatment of HIV / AIDS. Methodology: A literature review was conducted, with exploratory nature to deepen the knowledge on the theme worked in the research, seeking to provide greater familiarity with the problem. Results: The right to treat these people, as that of all citizens, is guaranteed by the Federal Constitution, being the responsibility of the Brazilian State. The emergence of new forms of treatment has greatly changed the disease landscape with decreased mortality, increased life expectancy of people living with AIDS. Conclusions: It is known that the new drugs for treatment have changed the history of the disease and today AIDS is treated in a controlled way and provides safety to the patient. It is possible to offer a combination of medicines for the treatment of the patient. harmony between the disease and the infected person. However, to achieve the benefits of treatment, it is necessary to use the medication correctly, both for initiation and withdrawal. Therefore, the pharmacist's role in caring for people with HIV is essential for successful treatment.

Key words:HIV; Medications; Treatment ; Pharmacist.

\section{Introdução}

O Vírus da Imunodeficiência Humana (HIV) é um retrovírus com genoma de RNA. Pertence ao grupo dos retrovírus citopáticos e não-oncogênicos e para multiplicar precisam de uma enzima chamada transcriptase reversa. Essa enzima é responsável pela transcrição do RNA viral para uma cópia de DNA que pode, então, integrar-se ao genoma do hospedeiro (BRASIL, 2010 a). O HIV, causador da Síndrome da Imunodeficiência Adquirida (AIDS), foi identificado no ano de 1981 e se tornou um marco na história da humanidade (ROMANELLI, 2006).

Estima-se que 36,7 milhões de pessoas em todo o mundo viviam com HIV em 2016, segundo o Programa Conjunto das Nações Unidas sobre HIV/AIDS. Desde 2010, as novas infecções por HIV entre os adultos caíram cerca de $10 \%$, de 1,9 milhões para 1,7 milhões. Em 2016, entretanto, em todo o mundo, mais de 1,8 milhões de pessoas foram infectadas pelo vírus. O Ministério da Saúde revelou que 827 mil pessoas vivem com HIV/AIDS (PROGRAMA CONJUNTO DAS NAÇÕES UNIDAS SOBRE HIV/AIDS - UNAIDS, 2017).

Para o tratamento da doença são usados coquetéis, chamados antiretrovirais que impedem a multiplicação do HIV e diminuem a quantidade do vírus no organismo. Com isso, as células de defesa do corpo podem melhorar a imunidade, diminuindo os riscos de desenvolver doenças oportunistas (BRASIL, 2010 a). 
A Lei Federal no 9.313, de 13 de novembro de 1996, uma conquista da sociedade brasileira, garantiu o acesso universal e gratuito ao tratamento antiretroviral no Brasil. A introdução dos anti-retrovirais, a partir da década de 90, e o surgimento contínuo de novas substâncias modificou o padrão da doença com a diminuição da mortalidade, aumento da sobrevida e melhoria da qualidade de vida das pessoas que vivem com HIV/AIDS (BRASIL, $2010 \mathrm{a}$ ).

A importância e complexidade do tratamento medicamentoso do portador de HIV/AIDS, assim como o dinamismo com que são incorporados novos fármacos durante o tratamento, dão ao farmacêutico um papel de destaque no apoio à qualidade da prescrição (ROMANELLI, 2006).

São muitas as drogas disponíveis para o tratamento do HIV/AIDS. Elas forneceram segurança ao portador, e fizeram da AIDS uma doença controlável. Entretanto, para se alcançar os benefícios do tratamento, é preciso o uso correto da medicação, tanto para início, como para a retirada da medicação. Portanto, o papel do farmacêutico na assistência a portadores de HIV/AIDS é essencial para o sucesso do tratamento (ZALESKI, 2008).

Para tanto, foi necessário realizar um estudo literário, com natureza exploratória para aprofundar os conhecimentos sobre a pesquisa, buscando em várias bases de dados, informações úteis para alcançar os objetivos propostos neste estudo.

\section{Metodologia}

Foi realizada uma revisão de literatura, com natureza exploratória para aprofundar os conhecimentos sobre o tema trabalhado na pesquisa, buscando proporcionar maior familiaridade com o problema.

Foi utilizado publicações pesquisadas nas bases de dado como SCIELO Scientific Electronic Library Online e Google Acadêmico, além de cartilhas e publicações do Ministério da Saúde. Foram usados para a construção do trabalho artigos, livros e publicações do Governo Federal para mostrar a importância da atuação do farmacêutico no acompanhamento do portador de AIDS. Para alcançar os objetivos propostos neste estudo, os descritores "HIV", "AIDS", "Tratamento da AIDS", "Farmacêutico na adesão ao tratamento da AIDS" ,sendo inclusos na 
pesquisa preferencialmente materiais de publicação nacional. A pesquisa teve início em março e se estendeu até outubro de 2018.

\section{O Vírus da Imunodeficiência Humana (HIV)}

O HIV, causador da AIDS, foi identificado no ano de 1981 e se tornou um marco na história da humanidade (VENTURA,2004).Os primeiros casos de AIDS surgiram nos Estados Unidos, e foram notificados pela imprensa como um novo mal que estaria se alastrando no âmbito da comunidade de homossexuais do país. Em 1982, com a verificação do mal também em cidadãos do Haiti e em pessoas hemofílicas, concluiu-se que o agente infeccioso estava ligado ao sangue e propôsse a sigla AIDS, para designar a doença. Em 1983, na França, ocorreu a identificação do vírus HIV como o agente causal e, no ano seguinte, houve a confirmação nos Estados Unidos (FERRAZ; CAMPOS, 2010).

Em 2016, 36,7 milhões de pessoas em todo o mundo viviam com HIV. Até junho de 2017, 20,9 milhões de pessoas tiveram acesso a terapia antirretroviral (UNAIDS, 2017). Segundo UNAIDS (2017), especialmente no Brasil, atualmente, aproximadamente 718 mil pessoas estão vivendo com HIV/AIDS, sendo que 574 mil foram diagnosticados, 313 mil estão em tratamento e apenas 236 mil apresentam carga viral indetectável.

O HIV é um retrovírus que, geralmente, se transmite pelas vias sexual, sanguínea e perinatal. A infecção viral apresenta quatro estágios básicos: incubação, infecção aguda, latência e AIDS. Trata-se de uma doença crônica, com evolução lenta. Durante o período entre a infecção pelo vírus e o aparecimento dos sinais clínicos da doença, o paciente é chamado soropositivo. A soropositividade apenas atesta a presença do vírus no organismo e não significa que o paciente esteja com AIDS (ZALESKI, 2008).

No início da epidemia, com a alta letalidade, que se expandiu de maneira dramática em todo mundo, a AIDS foi considerada uma doença grave e sinônimo de morte programada. A assistência medicamentosa disponível no momento para o tratamento era precária e ineficiente. Entretanto, após a descoberta das novas drogas de alta potência para o tratamento da doença, no final do século passado, houve profunda mudança na história natural da infecção pelo HIV (BRASIL, 2000). 
Dada a gravidade do problema em todo o mundo, nos meses seguintes à descrição do vírus, desenvolveram-se sucessivas ações nas áreas médica, de assistência social e de legislação profissional visando proporcionar uma melhoria da qualidade de vida dos pacientes, tendo sido de fundamental importância o início do emprego de medicamentos antirretrovirais (ARV), a partir de 1986, no tratamento dos pacientes soropositivos (EIDAM; LOPES; OLIEIRA, 2005).

Os ARV atuam restringindo a reprodução do HIV no sangue, sem eliminá-lo. Atualmente, são divididos em cinco classes ou grupos e, para combater o vírus, é necessário utilizar medicamentos de classes diferentes (coquetéis), sabendo-se que muitos pacientes tomam três a quatro antirretrovirais. A terapia desacelera a progressão da doença e, consequentemente, possibilita que o paciente usufrua de número variável de anos de sobrevida com uma qualidade de vida melhor (FERRAZ; CAMPOS, 2010).

Essa patologia é multifacetada e fatores de diferentes ordens produzem situações específicas no nosso país. Isto implica em constantes mudanças, que exigem rápida adaptação do Programa Nacional de Doenças Sexualmente Transmissíveis/Síndrome da Imunodeficiência Adquirida - PNDST/AIDS (FUNDAÇÃO OSVALDO CRUZ - FIOCRUZ, 2011). As manifestações clínicas podem demorar em torno de oito anos para se apresentarem e incluem doenças específicas e doenças sugestivas da imunodeficiência, dentre elas o sarcoma de Kaposi e a candidíase oral (BRITO; SOUZA; LUNA, 2006).

Entretanto, Ferraz e Campos (2010) advertem que, "embora o tratamento antirretroviral seja um esplêndido avanço, não livrou os pacientes HIV+ do preconceito de grande parte da comunidade". Tal discriminação, não justificada, leva muitos portadores de AIDS a desenvolver distúrbios psicoemocionais e a abandonar a terapia com ARV, aumentando as taxas de não-adesão. Neste último aspecto, da adesão ao tratamento, tem-se considerado que o farmacêutico pode desempenhar um importante papel, por vezes constituindo-se mesmo em peça-chave dentro da equipe assistencial dos pacientes soropositivos, o que será discutido mais adiante.

\section{Tratamento da patologia}

A introdução dos anti-retrovirais, a partir da década de 90 , e o surgimento contínuo de novas substâncias modificou o padrão da doença com a diminuição da 
mortalidade, aumento da sobrevida e melhoria da qualidade de vida das pessoas que vivem com AIDS.

Segundo o Ministério da Saúde (BRASIL, 2010 b) existem duas classes de drogas liberadas para o tratamento anti-HIV. São os inibidores da transcriptase reversa, com drogas que inibem a replicação do HIV, bloqueando a ação da enzima transcriptase reversa, que age convertendo o RNA viral em DNA (Tabela 1).

Tabela 1 - Medicamentos (nucleosídeos, não-nucleosídeos e nucleotídeos) usados para tratamento do HIV / AIDS no Brasil - 2019.

\begin{tabular}{|c|c|}
\hline Tipo de drogas / Forma & Dosagem e frequência \\
\hline \multicolumn{2}{|r|}{ Nucleosídeos } \\
\hline Zidovudina (AZT) / comprimido & $\begin{array}{l}100 \mathrm{mg} \text {, dose:100mg 5x/dia ou 200mg 3x/dia ou } \\
300 \mathrm{mg} 2 x / \text { dia }\end{array}$ \\
\hline Zidovudina (AZT) /injetável & frasco-ampola de $200 \mathrm{mg}$ \\
\hline Zidovudina (AZT) / solução oral & frasco de $2.000 \mathrm{mg} / 200 \mathrm{ml}$ \\
\hline Didanosina (ddl) comprimido & 25 e 100mg, dose: 125 a 200mg 2x/dia; \\
\hline Zalcitabina (ddC) / comprimido & 0,75mg, dose: $0,75 \mathrm{mg} 3 x /$ dia; \\
\hline Lamivudina (3TC) / comprimido & 150mg, dose: 150mg 2x/dia; \\
\hline Estavudina (d4T) / comprimido & 30 e 40mg, dose: 30 ou 40mg 2x/dia; \\
\hline Abacavir / comprimidos & 300 mg, dose: 300 mg 2x/dia. \\
\hline \multicolumn{2}{|r|}{ Não-nucleosídeos } \\
\hline Nevirapina / comprimido & 200 mg, dose: 200 mg 2x/dia; \\
\hline Delavirdina / comprimido & 100 mg, dose: 400 mg 3x/dia \\
\hline Efavirenz / comprimido & 200 mg, dose: 600 mg 1x/dia. \\
\hline \multicolumn{2}{|r|}{ Nucleotídeos } \\
\hline Adefovirdipivoxil: comprimido & 60 e 120 mg, dose: 60 ou 120 mg 1x/dia \\
\hline
\end{tabular}

Ainda têm-se os inibidores da protease, que são drogas que agem no último estágio da formação do HIV, impedindo a ação da enzima protease que é fundamental para a clivagem das cadeias proteicas produzidas pela célula infectada 
em proteínas virais estruturais e enzimas que formarão cada partícula do HIV (Tabela 2).

Tabela 2 - Medicamentos inibidores de protases usados para tratamento do HIV / AIDS no Brasil - 2019.

\begin{tabular}{l|l}
\multicolumn{1}{c|}{ Tipo de drogas / Forma } & \multicolumn{1}{c}{ Dosagem e frequência } \\
\hline Indinavir / comprimido & $400 \mathrm{mg}$, dose: $800 \mathrm{mg} \mathrm{3x/dia;}$ \\
\hline Ritonavir / comprimido & $100 \mathrm{mg}$, dose: $600 \mathrm{mg} 2 \times /$ dia; \\
\hline Saquinavir / comprimido & $200 \mathrm{mg}$, dose: $600 \mathrm{mg} \mathrm{3x/dia;}$ \\
\hline Nelfinavir / comprimido & $250 \mathrm{mg}$, dose: $750 \mathrm{mg} \mathrm{3x/dia;}$ \\
\hline Amprenavir / comprimido & $150 \mathrm{mg}$, dose: $1.200 \mathrm{mg} 2 \times$ dia. \\
\hline
\end{tabular}

Fonte: Brasil (2010) b.

Na terapia combinada é usado o tratamento anti-retroviral com associação de duas ou mais drogas da mesma classe farmacológica, por exemplo, dois análogos nucleosídeos, ou de classes diferentes, dois análogos nucleosídeos e um inibidor de protease (CARNEIRO, 2006).

Estudos multicêntricos demonstraram aumento na atividade anti-retroviral (elevação de linfócitos T-CD4+ e redução nos títulos plasmáticos de RNA-HIV) quando da associação de drogas, particularmente redução da replicação viral por potencializar efeito terapêutico ou por sinergismo de ação em sítios diferentes do ciclo de replicação viral. Outros estudos evidenciaram redução na emergência de cepas multiresistentes quando da utilização da terapêutica combinada (BRASIL, 2010 b).

Visto que a terapia anti-retroviral é uma área complexa, sujeita a constantes mudanças, as recomendações deverão ser revistas periodicamente, com o objetivo de incorporar novos conhecimentos gerados pelos ensaios clínicos (EIDAM; LOPES; OLIVEIRA, 2005).

Com a implementação do Sistema Logístico de Medicamentos da AIDS, que visa garantir a distribuição continuada dos anti-retrovirais, o protocolo de assistência farmacêutica em DST/ HIV/ AIDS do Ministério da Saúde expõe e exemplifica detalhes sobre a dispensação, aquisição dos medicamentos, recomendação de 
tratamento de infecções oportunistas, dentre outros assuntos referentes ao tratamento e acompanhamento do paciente. (BRASIL, 2010 a).

Os medicamentos fornecidos aos pacientes são adquiridos pelo Ministério da Saúde, repassados às secretarias estaduais de saúde que, por sua vez, encaminham às secretarias municipais de saúde que os distribuem às unidades responsáveis pela dispensação. A responsabilidade da aquisição e distribuição dos medicamentos para o tratamento das doenças sexualmente transmissíveis é dos municípios (EIDAM; LOPES; OLIVEIRA, 2005).

A importância e complexidade do tratamento medicamentoso, assim como o dinamismo com que são incorporados novos fármacos, dão ao farmacêutico um papel de destaque no apoio a qualidade da prescrição. Isso inclui orientações individuais aos pacientes sobre o uso do medicamento, supervisão e orientação sobre a adequação dos esquemas antirretrovirais, doses, posologias e interações, participação em grupos de adesão e controle de faltosos, além de aspectos clínicos envolvidos com o manejo, informação e notificação de efeitos adversos (BRASIL, 2010 b).

\section{O papel do farmacêutico na adesão ao tratamento do HIV/AIDS}

Sabe-se que o farmacêutico se responsabiliza pela interação com 0 paciente, tendo como ponto principal a correlação das necessidades do mesmo com os medicamentos (PEREIRA; FREITAS, 2008). Estudos sugerem que o papel do farmacêutico aumenta a adesão ao tratamento e proporciona benefícios clínicos (CODINA; DELGADO, 2001; VENTURA; ALÓS, 2004), além de sugerir aumento do vínculo do usuário com o serviço de saúde (SILVEIRA, 2009).

Por isso, o envolvimento do farmacêutico com as equipes clínicas em hospitais que cuidam de paciente portador do vírus do HIV tem sido descrito desde 1991 em países desenvolvidos. Este fato tem sido associado ao aumento crescente da adesão pelo paciente ao tratamento (SABERI et al., 2012).

Entre fevereiro de 2007 e março de 2008, o Departamento de DST, AIDS e Hepatites Virais, coordenou um grupo de trabalho formado por farmacêuticos vinculados às Unidades Dispensadoras de Medicamentos Antirretrovirais (UDM) e pela logística das coordenações de DST/AIDS, pesquisadores, médicos e outros profissionais e pessoas vivendo com HIV/AIDS (PVHA), representantes da 
sociedade civil. O Departamento de DST, AIDS e Hepatites Virais, reconhecendo a importância estratégica do papel do farmacêutico, particularmente na dispensação de antirretrovirais - ARV, constituiu esse grupo com objetivo de fornecer subsídios atualizados, aprimorando, dessa forma, a qualidade da atenção às PVHA no Brasil (BRASIL, 2010 a).

Um dos pontos centrais do Protocolo de Assistência Farmacêutica em DST/HIV/AIDS é estabelecer recomendações e fornecer informações que aumentem a qualidade da intervenção do dispensador, particularmente do farmacêutico, na oportunidade singular do contato com o usuário, melhorando com isso a adesão, a identificação precoce de efeitos adversos, a orientação ao usuário sobre os medicamentos e suas interações (BRASIL, 2010 b).

De acordo com Primo (2015), no campo da atenção às pessoas que utilizam tratamento antirretroviral, um dos pontos de contato mais importantes do sistema de saúde com os usuários é o momento da dispensação do tratamento. Esse encontro permite que os farmacêuticos não apenas orientem e repassem informações ao usuário, mas que o façam em um processo de troca.

Uma meta-análise publicada em 2012 por Saberi e colaboradores mostrou que entre 10 publicações em que o farmacêutico fazia o acompanhamento farmacoterapêutico do paciente, em uso de antirretroviral, todas elas demonstravam associação ao aumento da adesão ao tratamento.

A atuação do farmacêutico no tratamento tem o intuito de fortalecer e orientar os pacientes para o uso racional de antirretrovirais, harmonizando procedimentos desde a programação, passando pelo sistema de informações, até orientações e recomendações gerenciais e clínicas sobre as diversas condições que fazem parte do cotidiano das pessoas que vivem com HIV/AIDS (BRASIL, 2010 b).

Durante a dispensação, podem-se identificar as pessoas que necessitam de abordagem especial, de acordo com os fatores de risco e o histórico de utilização inadequada dos medicamentos. Nesses casos, pode-se encaminhar o usuário para o acompanhamento farmacêutico individual ou coletivo. $O$ acompanhamento e orientações do paciente envolvem um processo de escuta, individualizado ou coletivo, centrado nas necessidades do usuário. Pressupõe a capacidade de estabelecer uma relação de confiança entre a equipe e o paciente, para que ele mesmo tenha possibilidade de reconhecer-se como sujeito de sua própria saúde e transformação (NAVES; HAMANN; SILVER, 2005). 
Sabe-se que o farmacêutico tem a responsabilidade de acompanhar o paciente durante toda sua trajetória de uso do medicamento. Seu papel será de identificar os principais fatores de riscos para a não adesão, prestar informações sobre os medicamentos utilizados, identificar interações medicamentosas, acompanhar e estimular os pacientes a manejar o seu próprio tratamento (PRIMO, 2015).

Entretanto, esse conjunto de atividades somente tem sentido quando em consonância com as necessidades reais dos usuários últimos dessas ações, as pessoas vivendo com HIV/AIDS. Esse processo aponta para a construção de uma aliança estratégica entre as equipes de saúde e os usuários, objetivando, com isso, promover a melhora da qualidade de vida e fortalecer o impacto favorável do acesso universal ao tratamento antirretroviral nos indicadores de morbidade e mortalidade, que caracterizam a reconhecida resposta brasileira à epidemia (BRASIL, 2010 a).

\section{Resultados e discussão}

No Brasil, apesar de ter sido adotada uma política governamental de acesso universal e gratuito aos ARV, formalizada desde 1996, e da divulgação periódica de alguns dados estatísticos animadores pelo Ministério da Saúde, o cenário parece não diferir muito dos demais países, constituindo a não-adesão importante fator a limitar não só a plena recuperação de muitos pacientes como a própria busca da cura da doença (TEIXEIRA; PAIVA; SHIMA, 2000).

Apesar das diferenças em magnitude e tendências, o quadro de análises revelado no estudo de Guimarães et al. (2017) é preocupante. A pesquisa indica que, diferentemente do esperado, os coeficientes de mortalidade por HIV/AIDS no Brasil são heterogêneos e apresentam importante aumento nos anos estudados. Poucos locais tiveram redução, como São Paulo, Brasília, Santa Catarina, Minas Gerais e Goiás. O crescimento ocorreu, em sua maioria, nos estados das Regiões Norte e Nordeste.

Conforme visto, as novas drogas para tratamento do HIV/AIDS mudaram a história da doença, controlando a morbimortalidade e possibilitando um tratamento seguro e tolerável ao portador. Contudo, o paciente precisa aderir ao tratamento com ARV e buscar ajuda psicológica para enfrentar os problemas relacionados ao preconceito e tentar levar uma vida de qualidade (PRIMO, 2015). 
Sabendo-se que a promoção da adesão constitui um desafio, e que a falta de adesão não é uma característica das pessoas, mas uma condição dependente da modificação no estilo de vida e que exige acompanhamento, o farmacêutico juntamente com a equipe profissional precisa dar o suporte necessário para que 0 paciente não desista do tratamento (SIMONI et al., 2003).

São muitos os fatores que contribuem para o processo de não adesão ao tratamento e também o abandono, entre eles estão o contexto socioeconômico desfavorável, dificuldade de compreensão do tratamento prescrito devido a baixa condição sociocultural, não adaptação da terapia à rotina diária, depressão, uso de bebidas alcoólicas e drogas ilícitas, além de reações adversas ao medicamento (LIMA; MALTA, 2005).

Osterberg e Blaschke (2005) apontaram em seus estudos que as dificuldades mais enfrentadas para a não adesão estão sob o controle dos pacientes. Dentre elas estão o esquecimento $(30 \%)$, outras prioridades $(16 \%)$, decisão de pular doses (11\%) e fatores emocionais (7\%). Estudos feitos por Ceccato et al., (2004) mostram que de 30 a $50 \%$ dos pacientes em terapia antirretroviral apresentavam problemas com adesão por falta de informação acerca dos medicamentos. Esse declarou ainda que os pacientes possuíam a compreensão insuficiente sobre o uso do esquema de antirretrovirais, aliada à falta de conhecimento sobre os riscos da não adesão e estes fatores são preponderantes para a administração incorreta da terapia medicamentosa.

O farmacêutico pode acompanhar o paciente durante toda sua trajetória de uso do medicamento. Seu papel será de "identificar os principais fatores de riscos para a não adesão, prestar informações sobre os medicamentos utilizados, identificar interações medicamentosas, acompanhar e estimular os pacientes a manejar o seu próprio tratamento" (PRIMO, 2015).

Em um estudo longitudinal realizado por Vielmoet al., (2014) com adultos em início de terapia, foi visto que o acompanhamento farmacoterapêutico permitiu a análise de diferentes fatores relacionados ao tratamento ARV. Em meio a eles, a análise entre $\mathrm{o}$ autoconhecimento referido pelos pacientes sobre HIV/AIDS e tratamento e o conhecimento aferido após o acompanhamento com o farmacêutico, ocorrendo aumento da compreensão por parte dos pacientes em relação à sua condição e tratamento. Fato esse, que pode demonstrar a contribuição da atenção farmacêutica para o empoderamento dos sujeitos e que poderá influenciar 
positivamente na manutenção de taxas satisfatórias de adesão ao longo do tratamento, que até o presente momento é para toda a vida.

Sabe-se que, quanto maior é o número de medicamentos utilizados por um indivíduo, maior é o risco de ocorrência de interações medicamentosas, e elas ocorreram em $30 \%$ dos pacientes acompanhados nos estudos de Vielmoet al. (2014). No entanto esse autores ainda destacam que o farmacêutico pode contribuir na monitorização do tratamento, realizando seu papel no planejamento do cuidado de cada paciente, como parte de uma equipe multidisciplinar a fim de garantir um atendimento integral às PVHA que acessam a Unidade Dispensadora de Medicamentos (UDM).

A integração entre os diferentes profissionais que compõem a equipe que cuida do paciente permite a combinação de conhecimentos especializados e corresponsabilidade de forma a proporcionar o alcance de melhores resultados em termos de adesão. Nesta perspectiva, cabe a equipe de saúde identificar as dificuldades apresentadas pelo paciente, tirar dúvidas, bem como prestar orientações específicas sobre a doença e o tratamento. Neste contexto, entende-se que a inserção do farmacêutico à equipe multidisciplinar, vem trazendo benefícios e impactos positivos na adesão (PRIMO, 2015).

Assim, o acompanhamento farmacoterapêutico poderá auxiliar as equipes de saúde envolvidas no trabalho com HIV/AIDS em todo o período de tratamento, verificando possíveis efeitos adversos, interações medicamentosas, e, principalmente acompanhando a terapia com o esquema ARV mais adequado para o paciente.

\section{Considerações finais}

O avanço da medicina, a reorganização dos movimentos sociais e a reforma do setor de saúde permitiram que o Brasil ingressasse em um movimento de intensa reestruturação na área de saúde, que inclui além da distribuição de novas drogas, a formação e prática dos profissionais de saúde, visando o bem estar e qualidade de vida do paciente.

Considerando que o direito ao tratamento de saúde é garantido pela Constituição Federal a todos os cidadãos, o surgimento de novas formas de 
tratamento mudou muito o cenário da doença com a diminuição da mortalidade, aumento da expectativa de vida das pessoas que vivem com AIDS.

Entretanto, para se alcançar os benefícios do tratamento, é preciso o uso correto da medicação, tanto para início, como para a retirada da medicação. Uma clara conexão pode ser constituída entre a aplicação da terapia antirretroviral e a melhoria da qualidade de vida dos pacientes. Apesar de plausíveis efeitos indesejados, os ARV têm permitido melhoria dos pacientes não apenas em relação à infecção viral, mas também da depressão, ansiedade e outras alterações psicológicas que costumam acompanhar o portador de HIV.

Independente da condição econômica do país, a terapia antirretroviral precisa ser realizada em todos os soropositivos, e a não-adesão ao tratamento significa a perda da qualidade de vida e redução das chances de tratamento.

Portanto, o papel do farmacêutico na assistência à portadores de HIV é essencial para o sucesso do tratamento. Ressalta-se que a participação do farmacêutico no aumento da adesão desses pacientes tem sido reconhecida mundialmente. Contudo, há barreiras a superar nesse aspecto, sendo a falta de adequada qualificação profissional possivelmente a mais importante delas.

É possível concluir neste estudo que a adesão ao tratamento tende a aumentar quando a equipe multiprofissional conta com um farmacêutico que atua de forma clínica e não apenas na dispensação e nas tarefas administrativas da farmácia.

\section{Referências}

BRASIL, MINISTÉRIO DA SAÚDE. Secretaria de Vigilância em Saúde. Programa Nacional de DST e Aids. Recomendações para terapia antirretroviral em adultos infectados pelo HIV - 2000. 7. ed. Brasília, 2008. Serie Manuais, n. 2.

BRASIL, MINISTÉRIO DA SAÚDE. Secretaria de Vigilância em Saúde. Programa Nacional de DST e Aids. Diretrizes para o fortalecimento das ações de adesão ao tratamento para pessoas que vivem com HIV/Aids. Brasília, 2010 a.

BRASIL. Ministério da Saúde. Secretaria de Vigilância em Saúde. Programa Nacional de DST e Aids. Recomendações para Profilaxia da Transmissão Vertical do HIV e Terapia Antirretroviral em Gestantes: manual de bolso. Secretaria de Vigilância em Saúde, Programa Nacional de DST e Aids. - Brasília : Ministério da Saúde, 2010 b. 
BRITO, A. M; SOUZA, E.A; LUNA, C. L. Regional patterns of the temporal evolution of the aids epidemic in Brazil following the introduction of antiretroviral

therapy.BrazilianJournalofInfectiousDiseases, v.9, n.1, p. 9-19, 2006.

CARNEIRO, Sílvia de Andrade. Efeitos adversos da terapia antirretroviral. PósGraduação em Ciências da Saúde: Saúde da Criança e do Adolescente (UFMG). 2006. Disponível em:

http://www.smp.org.br/arquivos/site/pediatras/comunicados/efeitos-adversos-dosantirretrovirais-pdf.pdf. Acesso em 25 de outubro de 2018.

CODINA, C; DELGADO, O. Recomendaciones para desarrollarun programa de atenciónfarmacéuticaal paciente VIH. Comisión de normas y procedimientos de la SEFH. Espanha: SociedadEspañola de FarmaciaHospitalaria, 2001.

EIDAM, C.L.; LOPES, A.S.; OLIVEIRA, O.V. Prescrição de exercícios físicos para portadores do vírus HIV. Rev. Brasil. Ciência e Movimento, v.13, p.7-15, 2005.

FERRAZ, T. L. B. CAMPOS, E. L. V. HIV/AIDS: evolução histórica, aspectos psicoemocionais da convivência com a doença e a participação do farmacêutico na adesão ao tratamento. 8 Simposio de Ensino de Graduação. Mostra Acadêmica UNIMEP, 2010.

FIOCRUZ - FUNDAÇÃO OSVALDO CRUZ. Programa Nacional de Doenças Sexualmente Transmissíveis/Síndrome da imunodeficiência adquirida. Disponível em: http://www.ensp.fiocruz.br/portal-ensp/judicializacao/pdfs/515.pdf. Acesso em 22 de maio de 2019.

GUIMARÃES, M. D. C; CARNEIRO, M; ABREU, D. M. X; et al. Mortalidade por HIV/Aids no Brasil, 2000-2015: motivos para preocupação? RevBrasEpidemiol, vol. 01, n. 20,p,182-190, 2017.

LIMA, H. M. M.; MALTA, M. Guia para profissionais de saúde. Atendimento a pacientes HIV positivos usuários de drogas na rede pública de saúde. OPAS. Washington DC, 2005.

NAVES, J. O. S.; HAMANN, E. M.; SILVER, L. D. Orientação farmacêutica para DST: uma proposta de sistematização.Ciência e Saúde Coletiva, v. 10, n.4, p.10051014,Rio de Janeiro, Oct./Dec. 2005.

OSTERBEG, L.; BLASCHKE, T. Adherence to medication. The New England Journal of Medicine,v. 353, n.1, p. 487-497, 2005.

PEREIRA L. R. L; FREITAS O. A evolução da atenção farmacêutica e a perspectiva para o Brasil. Rev. Bras. Ci. Farm. Vol. 44 n. 4. p 601-612, 2008.

PRIMO, L. P. Gestão do cuidado em HIV/AIDS: impacto da atuação do farmacêutico clínico na adesão à Terapia Antirretroviral 21p. (TARV). Dissertação (Mestrado) apresentada à Faculdade de Medicina de Ribeirão Preto/USP. Orientador: Bollela, Valdes Roberto. 2015. 21p. 
ROMANELLI, R. M. Perfil das gestantes infectadas pelo HIV atendidas em pré-natal de alto risco de referência de Belo Horizonte. Rev. Bras. SaúdeMatern. Infant., Recife, v. 6, n. 3, p. 329-334, 2006.

SABERI, P. et al. The impact of HIV clinical pharmacist on HIV treatment outcomes: a systematic review.PatientpreferenceanAdherence, v. 6. n. 1. p. 297-322, 2012.

SILVEIRA M. P. T. Avaliação da efetividade da atenção farmacêutica sobre a adesão de pacientes HIV-positivos à terapia anti-retroviral. Dissertação.

(Mestrado em Medicina). Universidade Federal do Rio Grande do Sul, Porto Alegre, 2009.

SIMONI, J. M. et al. Antiretroviral adherence interventions: a review of current literature and ongoing studies. International Aids Society-USA, Topics in HIV Medicine, v. 11, n. 6, p. 185-198, 2003.

TEIXEIRA, P.R.; PAIVA, V.; SHIMA, E. Tá difícil engolir? Experiências de adesão ao tratamento anti-retroviral em São Paulo. São Paulo, Copidart, 2000.

UNAIDS - Programa Conjunto das Nações Unidas sobre HIV/AIDS. RESUMO INFORMATIVO - Dia Mundial Contra a AIDS 2017.

VENTURA C. J. M; ALÓS A. M. Programa de atenciónfarmacéutica a pacientes VIH com tratamiento antirretroviral: metodología y documentación. Farm.Hosp. (Madrid), v.28, n.1 p. 72- 9, 2004.

VIELMO, L. et al. Atenção farmacêutica na fase inicial de tratamento da AIDS como fator importante na adesão aos antirretrovirais. Rev. Bras. Farm, v. 95, n. 2, p.617635, 2014.

ZALESKI, E.G.F. O sentido de vida do portador da AIDS: uma questão de saúde mental. Campo Grande, Editora UFMS, 2008. 